\title{
Opinión y perspectiva de los productores de caña de azúcar del valle Grullo-Autlán, Jalisco, en relación a los apoyos de las organizaciones cañeras locales
}

\author{
Opinion and perspective of sugarcane producers of El Grullo-Autlán Valley, \\ Jalisco, in relation to local sugarcane organizations
}

Opinião e de perspectiva dos produtores de cana-de-açúcar Grullo-Autlan, Jalisco vale, em relação ao apoio de organizações locais cañeras

Sandoval-Legazpi, J. de J. ${ }^{1}$ Universidad de Guadalajara, México slegazpi@cucsur.udg.mx

B. L., Figueroa-Rangel ${ }^{2}$ Universidad de Guadalajara, México bfrangel@cucsur.udg.mx

J. J. Velázquez-Núñez ${ }^{3}$

Universidad de Guadalajara, México

jvelazque@cucsur.udg.mx

\section{Resumen}

Las actividades campesinas en el valle de El Grullo-Autlán son de gran importancia para el progreso y tranquilidad social de sus habitantes, ya que los cultivos básicos son parte importante en su economía y consumo. En las últimas décadas la caña de azúcar ha jugado un papel relevante en el desarrollo de esta región dada la gran importancia de este cultivo tanto a nivel nacional, como internacional y donde la demanda del mismo es parte

\footnotetext{
${ }^{1}$ Profesor-Investigador Titular. Departamento de Ecología. Centro Universitario de la Costa Sur. Universidad de Guadalajara.slegazpi@cucsur.udg.mx

${ }^{2}$ Profesor-Investigador Titular. Departamento de Ecología. Centro Universitario de la Costa Sur. Universidad de Guadalajara.bfrangel@cucsur.udg.mx

${ }^{3}$ Profesor-Investigador Asociado. Departamento de Contaduría Pública. Centro Universitario de la Costa Sur.

Universidad de Guadalajara. jvelazque@cucsur.udg.mx
} 
sustancial en la economía mundial. Esta actividad ha permitido detectar bajo la percepción de algunos actores (ejidatarios, ingenio, asociaciones e inclusive gobierno), situaciones que generan molestia en los mismos, malos entendidos en el accionar de la cadena productiva de la caña de azúcar reflejados principalmente en los costos de los insumos del cultivo. Uno de los propósitos de este estudio es dar a conocer el sentir y perspectiva de los productores de caña de azúcar en relación a las organizaciones cañeras locales (CNPR y CNC). Los resultados muestran como la mayoría de los productores de caña de azúcar confían en las organizaciones cañeras locales ya que reciben los apoyos técnicos y económicos de manera ágil y expedita, aun así una pequeña minoría muestra su inconformidad en cuanto a los apoyos económicos y lenta asesoría técnica, aunado a los cada vez más altos costos de los fertilizantes.

Palabras clave: productores, caña de azúcar, sentir, organización.

\section{Abstract}

Farming activities in the of El Grullo-Autlán Valley are of great importance for the progress and peace of mind of its inhabitants, as the staple crops are an important part in its economy and consumption. In recent decades, sugar cane has played an important role in the development of this region, given the great importance of this culture both nationally and internationally and where demand is a substantial part in the global economy. This activity has made it possible to detect under the perception of some actors (communal land holders, wit, associations and even Government), situations that generate nuisance, bad misunderstandings in the actions of the production chain of sugar cane mainly reflected in the costs of farming input supplies. One of the purposes of this study is to present the feeling and perspective of producers of cane sugar in relation to local sugarcane organizations (CNPR and CNC, by their name in Spanish). The results show how most of the sugar cane producers rely on local sugarcane organizations since they receive technical and economic support of agile and expeditious way, yet a small minority shows their dissatisfaction regarding the economic support and slow technical advice, coupled with the increasingly high costs of fertilizers.

Key words: producers, sugarcane, feel, organization. 


\section{Resumo}

Atividades de camponeses no vale de El Grullo-Autlan são de grande importância para o progresso e tranquilidade social dos seus habitantes, como culturas básicas são uma parte importante da sua economia e do consumo. Nas últimas décadas, a cana tem desempenhado um papel importante no desenvolvimento desta região, dada a importância desta cultura nacional e internacionalmente e onde a demanda por ele é uma parte importante na economia global. Esta actividade tem detectado sob a percepção de alguns atores (ejidatarios, sagacidade, associações e até mesmo do governo), situações que causam desconforto no mesmo, mal-entendidos nas ações da cadeia produtiva da cana de açúcar refletiu, principalmente, os custos insumos agrícolas. Um dos propósitos deste estudo é apresentar os sentimentos e perspectivas dos produtores de cana em relação às organizações cañeras locais (CNPR e CNC). Os resultados mostram que a maioria dos produtores de cana-de contar com organizações cañeras locais e para receber apoio técnico e financeiro ágil e forma expedita, ainda uma pequena minoria mostrar sua insatisfação em relação ao apoio económico e aconselhamento lento técnica, juntamente com os cada vez mais elevados custos dos fertilizantes.

Palavras-chave: produtores, cana-de-açúcar, sentimento, organização.

Fecha Recepción: Junio 2016 Fecha Aceptación: Diciembre 2016

\section{Introducción}

Las actividades agrícolas en el valle de El Grullo-Autlán son parte primordial en el desarrollo de esta región y donde los cultivas básicos juegan un papel relevante dentro de su economía. De estos sobresale el cultivo de la caña de azúcar el cual en las últimas décadas ha tenido un gran repunte debido principalmente a la gran demanda mundial del azúcar. Esta actividad ha permitido detectar bajo la percepción de algunos actores (ejidatarios, ingenio, asociaciones e inclusive gobierno) comentarios de molestia en el accionar de la cadena productiva de la caña de azúcar reflejado principalmente en los costos de los insumos del cultivo. Al respecto algunos productores se quejan de las 
Organizaciones Cañeras y señalan que "solo les roban el dinero de sus liquidaciones ya que en la zafra anterior el costo de gastos de organización alcanzó hasta los 70 pesos por tonelada",

Asimismo problemas en las calendarizaciones de riego y quema de la caña, alza en los insumos (principalmente en los fertilizantes), así como escasa asesoría en el manejo de sus tierras, son comentarios comunes en los pasillos y en algunas asambleas hechos por productores que muestran de alguna manera su inconformidad. Esto se pudiera ver reflejado en el funcionar de la industria azucarera local y más aún cuando en la región se encuentran dos asociaciones de cañeros, la Confederación Nacional de la Propiedad Rural (CNPR) y la Confederación Nacional Campesina (CNC), aunado al vaivén internacional de la oferta y demanda de este producto.

A este respecto Domínguez (2005) menciona que la oferta y demanda mundial del azúcar se ha visto empañada por diferentes situaciones, siendo una de ellas los excedentes en la producción de la cual se derivan dos vertientes, una es sobre la misma sobreproducción y la otra los cambios de hábitos de consumo o sustitución del azúcar por edulcorantes artificiales (jarabes de maíz de alta fructuosa, aspartato, sacarina, entre otros).

Además y con un gran impacto de inferencia, se presentan como tal los factores ambientales, los cuales son y serán un fuerte obstáculo para alcanzar una producción óptima para dar respuesta a la demanda de azúcar que existe actualmente, ya que a lo menos de manera local dicha problemática ambiental jugará un papel relevante en el futuro debido principalmente a la pérdida de estacionalidad de la lluvia, situación no ajena al contexto nacional e internacional.

\footnotetext{
${ }^{2}$ Melchor, H. (2016). Cede ingenio ante presión de cañeros. El mundo de Córdova. Tu mundo a diario. (11 de agosto de 2016).
} 
El presente trabajo muestra la opinión y perspectiva de los productores de caña de azúcar a los apoyos tanto técnicos, como económicos que les brindan las Organizaciones locales como lo son la Confederación Nacional de la Propiedad Rural (UNC-CNPR), la Confederación Nacional Campesina (CNC) y el Ingenio Melchor Ocampo (IMO).

\section{La dinámica de la producción de la caña de azúcar mundial y nacional}

El accionar de la caña de azúcar a nivel mundial ha permeado tanto la producción local como la regional. Sin embargo es la producción de azúcar a nivel global la que determina los vaivenes de demanda y oferta de este endulzante. Dicha producción mundial ha tenido en los últimos 5 años un ligero repunte, lo que ha generado que aparentemente el mercado del azúcar se estabilice. Sin embargo si a esto se le agrega la producción de caña de azúcar para otros fines como es la producción de biocombustibles (etanol), dicho excedente se podría ver alterado, por lo que se necesitará de más producción de azúcar en un futuro para cubrir la demanda existente tanto para consumo, como para la generación de dichos biocombustibles.

Al respecto datos recientes señalan como en los últimos 5 años la producción de azúcar apenas ha cubierto la demanda de consumo (Tabla 1).

Tabla 1. Balances mundiales de azúcar (Octubre-Septiembre 2014-2015)

\begin{tabular}{|c|c|c|c|c|c|c|c|}
\hline \multicolumn{8}{|c|}{$\begin{array}{l}\text { BALANCES MUNDIALES DE AZÚCAR (Octubre/Septiembre) } \\
\text { En miles de toneladas, Tel quel }\end{array}$} \\
\hline & $2014 / 15$ & $2013 / 14$ & $2012 / 13$ & $2011 / 12$ & $2010 / 11$ & $2009 / 10$ & 2008/09 \\
\hline Producción & 172,083 & 171,002 & 172,029 & 164,629 & 154,927 & 149,333 & 140,818 \\
\hline Consumo & 171,463 & 168,368 & 164,292 & 158,060 & 153,254 & 152,122 & 151,626 \\
\hline Superávit/déficit & 620 & 2,634 & 7,737 & 6,569 & 1,673 & 2,789 & 10,808 \\
\hline Demanda de importaciones & 55,720 & 56,390 & 60,533 & 54,325 & 53,870 & 53,991 & 48,395 \\
\hline Disponibilidad de exportaciones & 56,036 & 56,366 & 60,530 & 54,322 & 53,867 & 53,997 & 48,390 \\
\hline Inventario Final & 79,885 & 79,581 & 76,923 & 69,326 & 62,754 & 61,078 & 63,873 \\
\hline Inventario/Consumo & 46.59 & 47.27 & 46.82 & 43.86 & 40.95 & 40.15 & 42.13 \\
\hline
\end{tabular}

Fuente: ISO. Quarterly Market Outlook -February, 2015, (citado por CONADESUCA, 2015). 
Por otra parte los fenómenos meteorológicos han jugado un papel importante, tal es el caso de la dinámica de la lluvia al presentar años con buenos temporales y otros no tan buenos, situación que se ha reflejado en los últimos cinco años con un ligero aumento de la lluvia, lo que ha llevado a fluctuaciones productivas de la caña de azúcar, incidiendo con ello que la oferta en ocasiones sea un poco mayor que la demanda y viceversa, aunado esto a la producción de los biocombustibles.

México no ha estado ajeno a esto vaivenes mundiales tanto del clima, como de la producción de azúcar. En datos recientes para nuestro país, donde la situación no es nada halagüeña dados los costos de arancel los cuales se vieron reducidos en las importaciones hacia los Estados Unidos. En el ciclo 2013-2014 dichas importaciones se redujeron en 96.000 toneladas métricas (TM), las cuales fueron destinadas al Programa de reexportación de México (IMMEX) para los productos que contienen azúcar. Las entregas para el consumo se redujeron en 50.000 MT después de una caída en los envíos nacionales de fin de temporada. Las entregas totales por lo tanto se redujeron en 156.000 TM (Riche, et-al, 2014).

La misma fuente cita que para el periodo 2014-2015 la situación no fue nada mejor, ya que para este periodo, la oferta total de México se incrementó en 71.000 toneladas en las reservas iniciales. La entrega para el consumo se redujo en 52.000 TM en línea por la reducción hecha en el 2013/14. Las reservas finales se pronostican en un $22 \%$ del consumo con una reducción de 11,000 TM a 936,000 TM. Por otra parte esa merma de azúcar a nivel nacional ha generado que los precios de este producto se incremente año con año. Así lo señala ZAFRANET (2015), quien comenta como dicho precios en los bultos de $50 \mathrm{Kg}$. han ido a la alza en los últimos cuatro meses (Gráfica 1).

En dicha gráfica se muestra como los precios de la azúcar se mantuvieron con cierta estabilidad del octubre de 2014 a junio de 2015, donde después de esta fecha los precios se incrementaron en más del $50 \%$. 
Gráfica 1. Pesos por bulto de $50 \mathrm{Kg}$ en azúcar estándar en los últimos 12 meses

(Oct 2014-Oct 2015).

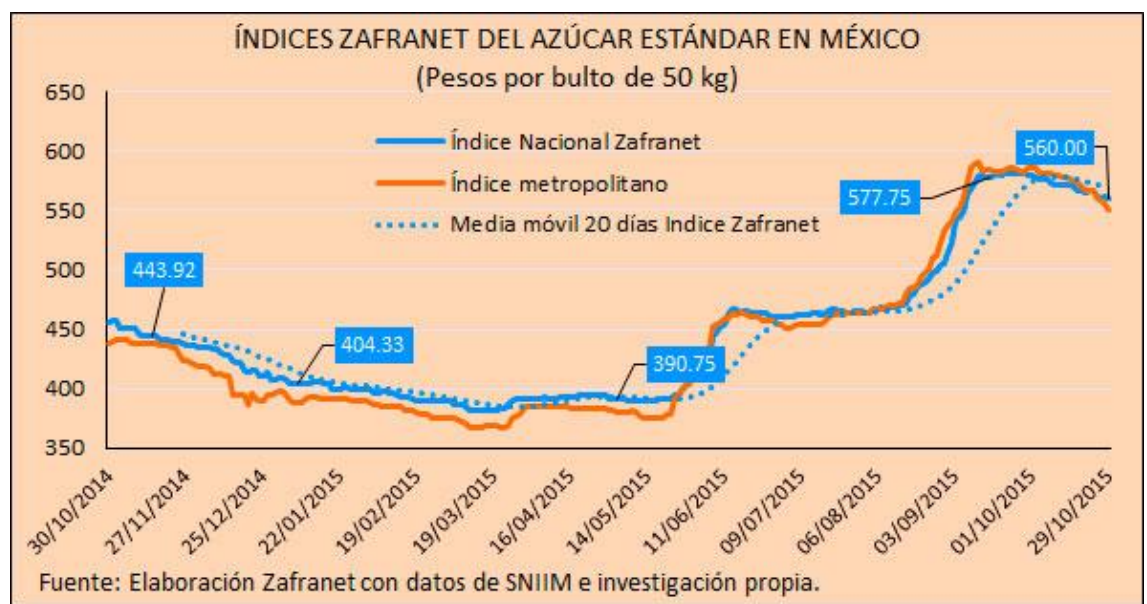

Fuente ZAFRANET (2015). (Consultado el 30 de Octubre de 2015)

Esta situación no ha sido ajena al valle de El Grullo-Autlán, que con su dinámica agrícola representa una de sus principales actividades económicas más importantes, donde se destaca la producción de cultivos básicos (fríjol, maíz, sorgo y arroz), hortalizas (melón, sandía, calabacita, tomate de cáscara, chile y jitomate) y cultivos frutales (naranja, lima y limón) y perennes como alfalfa, caña de azúcar y agave en los últimos años (Quintero, 2003).

Sin embargo es la caña de azúcar la que ha tomado gran relevancia en estas últimas décadas, debido a su importancia económica y estratégica para el desarrollo regional, dadas las extensas superficies de suelos dedicadas para su cultivo, así como a la generación de empleos e ingreso y sobre todo a la importancia del mercado azucarero. Esta dinámica azucarera ha permitido detectar bajo la percepción de los productores de caña de azúcar una serie de preocupaciones, donde sobresalen mediante sus comentarios, lo caro de los insumos para su cultivo, señalando a los fertilizantes y a sus tierras como su mayor preocupación, ya que según ellos año con año se van cansando. Esto genera en ellos inquietudes y malos entendidos que se pudieran ver reflejados en el funcionar de la industria azucarera regional. 


\section{Problemas actuales a que se enfrentan los productores de caña de azúcar a nivel nacional}

Dentro de los problemas actuales a que se enfrentan los productores de caña de azúcar a nivel nacional, están aquellos relacionados a los pagos tardíos de su cosecha. Melchor (2016, 11 de agosto), señala que los cañeros adheridos a la CNC y CNPR del ingenio Central Progreso, mostraron su inconformidad en basa al incumplimiento de pago de la liquidación final de la zafra 2015-2016 que sería a 6 semanas y quería aplazar el pago a 8 semanas, por lo que amenazaron con bloquear el acceso a los trabajadores. Ellos señalan: "Estamos a las vueltas que esta semana y así nos llevan, primero que el pago sería por monto, luego por clave y al final que por zona y es la hora que no recibimos mi dinero, lo que atrasa los trabajos para la próxima cosecha". Otro señaló: "Nosotros estamos cumpliendo con los lineamientos del ingenio que exige calidad de la caña, con el retraso no podemos beneficiar las cañas como debe ser, nos falta el recurso", quien agregó que de acuerdo a la Ley Sustentable de la Caña de Azúcar, la liquidación debe realizarse a los 30 días de haber concluido zafra. "Que nos paguen a tiempo cada zafra es lo mismo y no es justo porque nosotros ya entregamos la gramínea", señaló otro productor.

Aunado a lo anterior otro problema que enfrentan también los productores es lo referente a la cartera vencida, aún y a pesar del incremento en la tonelada de azúcar para la zafra 2015- 2016. Ya que para el caso de los productores abastecedores del Ingenio "El Potrero", esto no fue suficiente ya que por su cartera vencida no reciben alcances de las últimas tres zafras. Así lo manifiesta un productor de éste Ingenio al señalar: "Al banco le pagamos el 10 por ciento mensual si son 100 mil son 10 mil mensuales de intereses". "En dos años de sequía se perdió la caña, nos quedamos sin caña sólo 50 toneladas metimos en 2012-2013”, expresó otro productor. Otro señaló “Los cañeros pagamos la reparación de caminos y seguro para los cortadores y sin alcances estamos en la quiebra" (Melchor, 2016, 19 de agosto). 
Asimismo otra gran problemática es la importación de alta fructuosa. Así lo señala el Ing. Carlos Blackaller Ayala, presidente de la Unión Nacional de Cañeros (UNC), quien define así el trasfondo de la problemática: "que la Secretaría de Economía deje de importar alta fructosa y que, al bloquear las bodegas de azúcar, automáticamente incremente el precio del endulzante”. Así lo manifestaron también los productores en variadas ocasiones por la excesiva importación de fructosa de maíz la cual asciende a 2 millones de toneladas (Howard 2013).

Finalmente esta serie de problemáticas son las que a través de la última década han afectado a los productores de caña de azúcar del país, lo que no excluye al valle de El Grullo-Autlán. Problemas que aún subsisten y que algunos agravan aún más día con día, como son las importaciones de alta fructuosa que sigue golpeando fuertemente al campo cañero mexicano, así como las carteras vencidas para algunos productores generadas por un mal temporal, situación común ya en nuestro campo mexicano.

\section{Metodología}

Se realizó una serie de encuestas para detectar la problemática anunciada vía oral por algunos productores de caña de azúcar mediante la asistencia a las Asambleas del gremio cañero. Siendo los puntos de delimitación los productores de caña de azúcar de las dos principales confederaciones de esta región. La Confederación Nacional de la Propiedad Rural (CNPR) y la Confederación Nacional Campesina (CNC), las cuales presentaron un padrón de agremiados de 1700, en una superficie dedicada al cultivo de caña de 12,000 ha. (CNPR, 2014).

En primera instancia y como parte de los recorridos de campo, se realizó dicho sondeo mediante entrevistas verbales a los dueños de las parcelas en cuanto a sus necesidades de asesoría, manejo, créditos a tiempo, riegos oportunos y la compra y aplicación adecuada de los fertilizantes. Además se aplicaron encuestas (10 preguntas consideradas clave) a productores de caña de azúcar pertenecientes al padrón de agremiados de estas asociaciones con la finalidad de detectar sus impresiones en cuanto al 
manejo de la caña de azúcar en sus parcelas. Además se tomaron en cuenta algunos comentarios adicionales que realizaron los mismos a través de cada una de las hojas de la encuesta, vislumbrando la problemática a estudiar.

Para el cálculo del tamaño de muestra (No. de entrevistas), ésta se realizó mediante la fórmula de muestras finitas (Santos et al., 2003) ${ }^{\text {a }}$, dado que se conocía el universo a encuestar. Se graficó en primera instancia cada uno de los cuestionamientos con la finalidad de observar la frecuencia o recurrencia de respuestas (Malhotra, 2008).

Donde:

$$
n=Z^{2}{ }_{\alpha} \frac{N}{i^{2}(N-1)+Z_{\alpha}^{2} \cdot p \cdot q}
$$

$\mathrm{n}=$ Tamaño de la muestra

$\mathrm{N}=$ Tamaño de la población (entrevistados potenciales)

$\mathrm{Z}=$ Valor correspondiente a la distribución de Gaus 1,96 para a $=0,05$ y 2,58 para a $=0,01$

$\mathrm{p}=$ Prevalencia esperada del parámetro a evaluar.

$\mathrm{q}=1-\mathrm{p}($ Si $\mathrm{p}=30 \%, \mathrm{q}=70 \%)$

$\mathrm{i}=$ Error que se prevé cometer. Por ejemplo, para un error del 10\%, introduciremos en la fórmula el valor 0,1. Así, con un error del 10\%, si el parámetro estimado resulta del 80\%, tendríamos una seguridad del 95\% (para a $=0,05$ ) de que el parámetro real se sitúa entre el $70 \%$ y el $90 \%$. Vemos, por tanto, que la amplitud total del intervalo es el doble del error que introducimos en la fórmula

La información fue sistematizada y ordenada para luego aplicar el análisis estadístico de las respuestas proporcionadas por los productores, tomando en cuenta los 9 de los 10 cuestionamientos, ya que la pregunta 5 no entró al análisis estadístico por ser una pregunta abierta. Resultando un total de 261 entrevistas, con un margen de confianza del $90 \%$.

Las respuestas de los 9 cuestionamientos fueron analizadas en el programa Stadistical Package for Social Science (SPSS) en su versión 15.0, y junto con las entrevistas verbales y los análisis estadísticos permitieron determinar las relaciones con respecto a las respuestas proporcionadas. 


\section{Resultados}

Los siguientes puntos abordan de manera gráfica, descriptiva y estadística la detección de la problemática mediante el sentir de los productores de caña de azúcar del valle de El Grullo-Autlán.

Análisis gráfico descriptivo

¿Por qué sembrar caña de azúcar y no otros cultivos como maíz, trigo, sorgo, etc.? (P1)

Respecto a esta pregunta, se dieron 5 opciones de respuesta, siendo estas: 0) Sin respuesta; 1) Deja más (\$) la caña; 2) Nunca he sembrado otro cultivo que no sea caña; 3) Para caña si me apoyan para sembrar; 4) Aquí se presta más para la caña. Al respecto la gráfica muestra como el productor de caña de azúcar señala su sentir y predilección por sembrar este cultivo al señalar que les deja más que otros, aunado a que se sienten de cierta manera “cobijados" por el Ingenio "Melchor Ocampo", indicando para ello, que para este cultivo "sí se les apoya" con más facilidad que para otros. (Gráfica 2).

Gráfica 2. Respuestas de los productores de caña de azúcar del valle de El Grullo-Autlán a la pregunta: ¿Por qué sembrar caña de azúcar y no otros cultivos como maíz, trigo, sorgo, etc.? (p1)

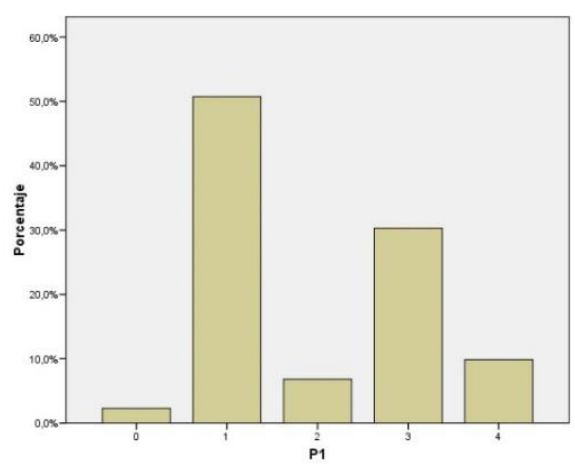


En la gráfica anterior (2), se puede observar como más del 50\% de los encuestados señaló que el cultivo de la caña de azúcar les deja más (económicamente hablando), aunado a que poco más del 30\% manifestó que los apoyos para la siembra de este cultivo son más fluidos al apoyárseles más.

¿El problema de mi caña es...? (P2)

Este cuestionamiento se realizó con base a 5 tipos de respuesta: 1) Fertilizante a tiempo; 2) Fertilizante caro; 3) Agua a tiempo (riego); 4) No me asesoran y 5) Mi suelo está agotado); 6) Más de una respuesta y 7) Más de dos respuestas, con la finalidad de rescatar la problemática planteada con anterioridad por vía verbal en cuanto a algunos problemas con el manejo y producción de la caña de azúcar. Los resultados muestran como los productores de caña de azúcar manifiestan que uno de los principales problemas al momento de preparar su parcela es el fertilizante, ya que este es demasiado caro lo que reduce en gran medida la posible ganancia que se pudiera tener (Gráfica 3).

Aunado a que consideran que una de las principales disminuciones en la producción de la caña de azúcar es porque sus suelos están agotados, situación que de gran manera se relaciona con el manejo de los mismos y una posible aplicación de abonos orgánicos como alternativa para enriquecer los suelos de sus parcelas y mitigar los costos del fertilizante.

Gráfica 3. Respuestas de los productores de caña de azúcar del valle de El Grullo-Autlán a la pregunta: El problema de mi caña es... (P2)

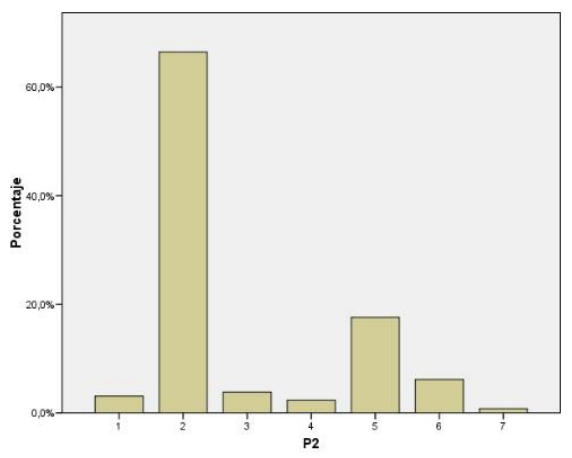


Más del $60 \%$ de los encuestados señala que los fertilizantes han ido a la alza en los últimos años, aunado a que cerca del $20 \%$ están preocupados porque sus suelos se agotan cosecha tras cosecha (Gráfica 3).

¿La calendarización de los riegos para su parcela son los adecuados? (P3)

Para esta preguntas se utilizó el siguiente tipo de respuestas: 1) Totalmente de acuerdo; 2) De acuerdo; 3 ) Indiferente; 4) En desacuerdo y 5) Totalmente en desacuerdo. Al respecto más de la mitad de los encuestados señaló que no hay problema con la calendarización de riego, a esto se suma que otra buena parte de los mismos manifestó que estaban bien, por lo que se puede afirmar que la mayoría de los productores están de acuerdo en dicho calendarios de riego, esto es, que la disponibilidad de agua es la adecuada (Gráfica 4).

Sin embargo cabe señalar que una mínima parte manifestaron inconformidad con la calendarización de riego, por lo que sería bueno investigar que está pasando con esta gente para aminorar su problema y darle una posible solución.

Gráfica 4. Respuestas de los productores de caña de azúcar del valle de El GrulloAutlán a la pregunta: ¿La calendarización de los riegos para su parcela son los adecuados? (P3)

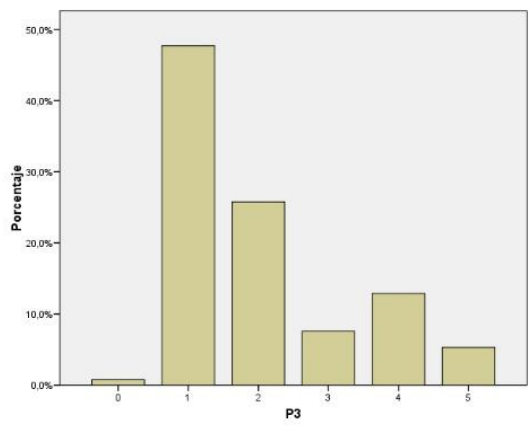

Alrededor del 50\% de los encuestados señaló que no tenían problema alguno con la calendarización de riego, ya que la misma les dejaba totalmente satisfechos, pero si a esto le agregamos que otro $25 \%$ señaló que estaban satisfechos, se puede afirmar que cerca del 
$80 \%$ de los encuestados no tienen problema con esta labor agrícola. Sin embargo es bueno acotar que poco más del 10\% muestra una inconformidad con los mismos (Gráfica 3).

¿Tiene problemas para el financiamiento de su cultivo? (P4)

Al respecto se dieron como opción 3 tipos de respuesta: 1) Sí; 2) No y 3) Más o menos. La mayoría de los entrevistados señaló que no tienen problema a esta parte económica, sin embargo existe una pequeña minoría que manifiesta si tener esta preocupación (Gráfica 5). Al respecto una minoría de los productores manifiestan:

Los insumos están bien caros y los gastos de organizaciones también, entonces no nos queda nada ${ }^{3}$

Los créditos nunca llegan a tiempo y jinetean el dinero ${ }^{4}$

El precio es barato y apenas sale para los fertilizantes ${ }^{5}$

Gráfica 5. Respuestas de los productores de caña de azúcar del valle de El Grullo-Autlán a la pregunta: ¿Tiene problemas para el financiamiento de su cultivo? (P4)

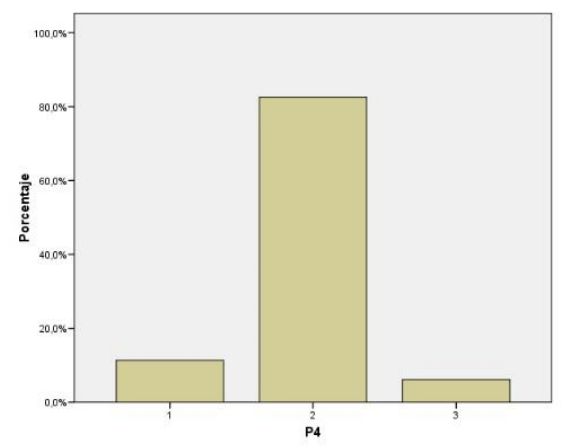

Son innegable algunas inconformidades mostradas. Sin embargo más del $80 \%$ de los entrevistados señala el no tener problemas con el financiamiento para su cultivo. Por otra parte existe un 10\% que no está de acuerdo con dicha situación (Gráfica 5).

\footnotetext{
${ }^{3}$ Entrevista No. 2 según sistematización de datos.

${ }^{4}$ Entrevista No. 20 según sistematización de datos.

${ }^{5}$ Entrevista No. 122 según sistematización de datos.
} 
¿Quién le otorga el financiamiento? Y ¿Cuál otorga el mejor financiamiento? (P 6 y 7)

Respecto a estas dos preguntas (6 y 7 de la encuesta), cuyas opciones de respuesta fueron: 0) Sin respuesta; 1) Ingenio; 2) Caja Popular; 3) Banco y 4) Particular: las respuestas señalan como más de la mitad considera que el Ingenio "Melchor Ocampo" mediante las Asociaciones Cañeras, otorgan el financiamiento y que dicha organización maneja las mejores opciones de crédito. Sin embargo la Caja Popular regional es otra muy buena opción para este fin, teniendo como tercera opción la vía particular.

Gráfica 6 y 7. Respuestas de los productores de caña de azúcar del valle de El GrulloAutlán a las preguntas: ¿Quién le otorga el financiamiento? (P6) Y ¿Cuál otorga el mejor financiamiento? (P7).
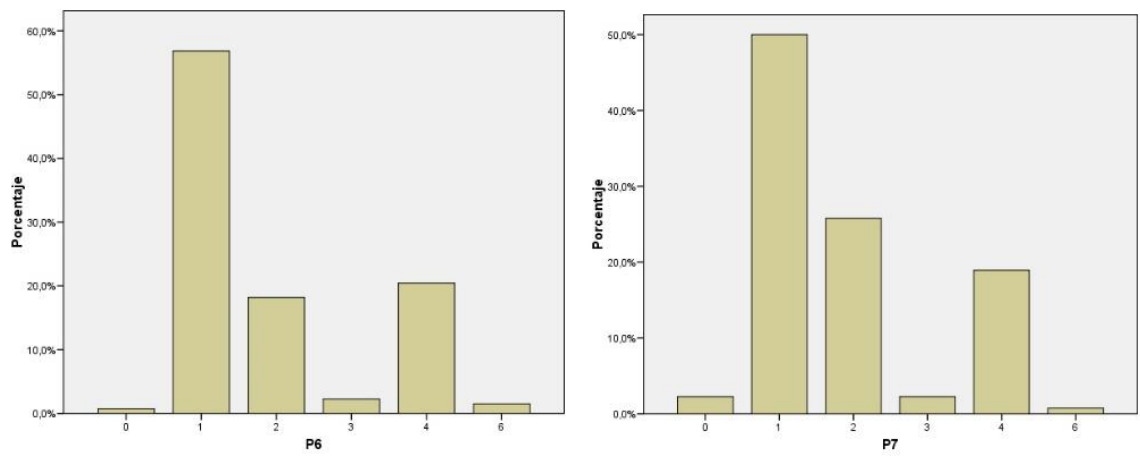

El Ingenio es considerado en más del 50\% como la mejor opción para otorgar financiamiento, así como el mejor financiamiento. Siendo la Caja Popular regional la otra opción con cerca del 30\% (Gráficas 6 y 7).

Con base a la respuesta anterior ¿Por qué considera que esta sea la mejor fuente de financiamiento? (P8)

Al respecto se les dio opción con 5 tipos de respuesta: 0) Sin respuesta; 1) Porque me cobra menos interés; 2) Porque me otorga el crédito más fácil; 3) Porque no me molesta tanto aun debiéndole poco y 4) Porque es la mejor. El sentir de los entrevistados fue contundente al señalar que los intereses son más bajos con los préstamos del Ingenio, aunado a que les 
facilitan el crédito y considerando que es la mejor opción.

Gráfica 8. Respuestas de los productores de caña de azúcar del valle de El Grullo-Autlán con base a la pregunta: ¿Por qué considera que esta sea la mejor fuente de financiamiento? (P8)

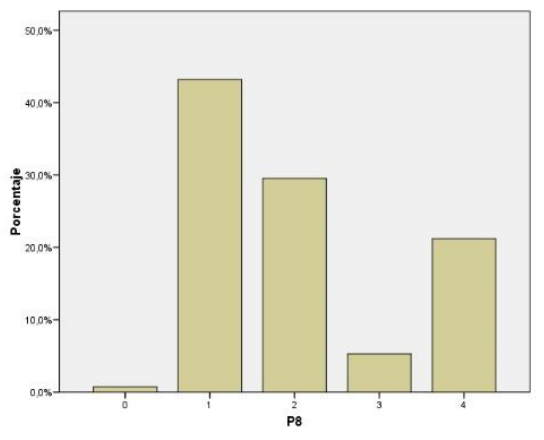

Más del 40\% de los encuestados considera el apoyo del Ingenio "Melchor Ocampo" como algo fundamental para el cultivo de su caña, ya que les cobra menos interés, además de darles el crédito con más facilidad (30\%). Aunado a que piensan que es la mejor opción (20\%) (Gráfica 8).

¿El apoyo de las Asociaciones Cañeras es importante para la siembra de su caña? (P 9)

A este cuestionamiento cuyas opciones de respuesta fueron: 1) Totalmente de acuerdo; 2) De acuerdo; 3) Indiferente; 4) En desacuerdo y 5) Totalmente en desacuerdo. El grueso de las respuestas señaló que el apoyo de las Asociaciones Cañeras es muy importante para la siembra de su caña. 
Gráfica 9. Respuestas de los productores de caña de azúcar del valle de El Grullo-Autlán a la pregunta: ¿El apoyo de las Asociaciones Cañeras es importante para la siembra de su caña? (P9)

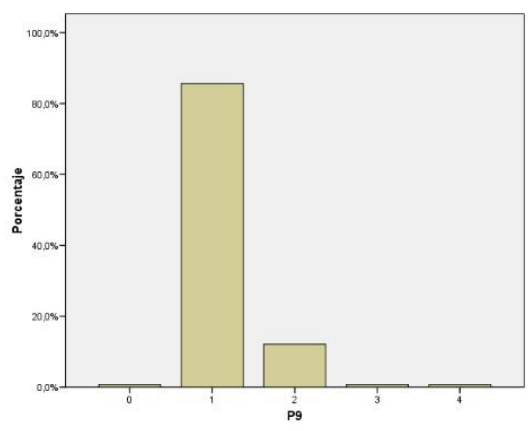

Más del 80\% de los encuestados señala que el apoyo de dichas Asociaciones ha sido un factor importante en la siembra de su caña, pero si a esto le sumamos el otro $15 \%$ de los que están de acuerdo, entonces se puede afirmar que el 100\% afirma que las Asociaciones son un factor importante en el cultivo de la caña de esta región (Gráfica 9).

¿Recibe a tiempo los apoyos de asesoría agrícola por parte de las Asociaciones? (P10)

Con base a las respuestas de apoyo: 1) Totalmente de acuerdo; 2) De acuerdo; 3) Indiferente; 4) En desacuerdo y 5) Totalmente en desacuerdo. Los encuestados señalaron en su mayoría que si reciben a tiempo los apoyos por parte de las Asociaciones. Si a esto sumamos que cerca de una séptima parte también está de acuerdo en dicho apoyo, la conformidad aumenta aún más por parte de los productores de caña de este valle. 
Gráfica 10. Respuestas de los productores de caña de azúcar del valle de El Grullo-Autlán a la pregunta: ¿Recibe a tiempo los apoyos de asesoría agrícola por parte de las

\section{Asociaciones? (P10)}

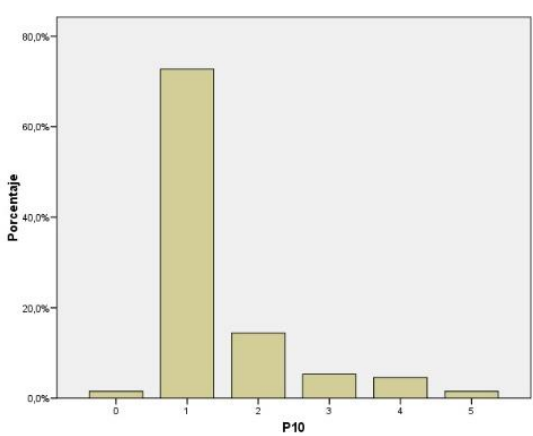

Se puede observar como más del $70 \%$ de los encuestados señala el estar completamente de acuerdo al recibir a tiempo los apoyos por parte de las Asociaciones Cañeras, lo que sumado al $15 \%$ de los que están de acuerdo, el nivel de confianza de los productores a estas Asociaciones se eleva a más del 80\% (Gráfica 10).

\section{Análisis estadístico}

El siguiente punto aborda el análisis estadístico de los datos, así como algunos comentarios adicionales realizados por algunos de los productores. Al respecto se aplicó la prueba no-paramétrica chi-cuadrada $\left(X^{2}\right)$ con la finalidad de detectar el nivel de confianza de las respuestas de los entrevistados. Dicho análisis reveló diferencias estadísticamente significativas $(p<0.05)$ en las respuestas de los productores a los cuestionamientos elaborados (Tabla 2).

Tabla 2. Resultados estadísticos $\left(\right.$ Chi-cuadrada $\left.X^{2}\right)$ de las respuestas proporcionadas por los productores de caña de azúcar del valle de El Grullo-Autlán

\begin{tabular}{lccccccccc}
\hline & P1 & P2 & P3 & P4 & P6 & P7 & P8 & P9 & P10 \\
\hline Chi- & & & & & & & & & \\
cuadrado(a,b,c & 108,455 & 307,863 & 120,909 & 144,591 & 183,636 & 147,818 & 80,273 & 361,485 & 307,545 \\
,d) & & & & & & & & & \\
Gl & 4 & 6 & 5 & 2 & 5 & 5 & 4 & 4 & 5 \\
Sig. asintót. & $\mathbf{0 . 0 0 1}$ & $\mathbf{0 . 0 0 1}$ & $\mathbf{0 . 0 0 1}$ & $\mathbf{0 . 0 0 1}$ & $\mathbf{0 . 0 0 1}$ & $\mathbf{0 . 0 0 1}$ & $\mathbf{0 . 0 0 1}$ & $\mathbf{0 . 0 0 1}$ & $\mathbf{0 . 0 0 1}$ \\
\hline
\end{tabular}


La tabla anterior muestra el nivel de confianza en cuanto a las respuestas de los productores entrevistados. Con base a dicho análisis se puede señalar que los productores de caña de azúcar del valle de El Grullo-Autlán siembran este cultivo porque desde su perspectiva les deja más que otros cultivos, no batallan con los apoyos, aunado a que su producto tiene el mercado directo mediante la molienda en el Ingenio y bajo un acuerdo de sus agremiados.

Sin embargo al preguntárseles ¿cuál era el problema de su caña?, mencionan que el fertilizante, ya que cada día es más caro para ellos, aunado que otra buena parte señaló que sus suelos estaban agotándose cosecha tras cosecha. Situación que lleva a indagar con este trabajo dicha problemática, quizás no tanto con los costos de fertilizantes, sino de observar en primera instancia que está pasando con sus suelos mediante estudios alternados de este tipo (quizás cada cinco años), así como una asesoría sobre el uso de abonos orgánicos para mitigar de alguna manera y a largo plazo el uso continuo de fertilizantes, los cuales se manejarían como un complemento y no como una sustitución de uno hacia el otro (Sandoval y Sepúlveda, 2012). Pero es bueno mencionar que algunos productores entrevistados (una minoría), manifestaron molestias dentro de la dinámica agrícola de este valle con estas Asociaciones Cañeras. Al respecto y en relación con la pregunta No. 5: ¿Tiene problemas para el financiamiento de su cultivo?, la cual no entró en el proceso de análisis estadístico, dado que era una pregunta abierta, se tiene lo siguiente (Tabla 3): 
Tabla 3. Respuestas proporcionadas por los productores de caña de azúcar del valle de El Grullo-Autlán a la pregunta 5: ¿Tiene problemas para el financiamiento de su cultivo?

\begin{tabular}{|c|c|}
\hline $\begin{array}{l}\text { No. De encuesta de acuerdo a } \\
\text { la sistematización de los datos }\end{array}$ & Respuesta del encuestado \\
\hline 2 & $\begin{array}{l}\text { "Los insumos están bien caros y los gastos de organizaciones } \\
\text { también, entonces no nos queda nada" }\end{array}$ \\
\hline 8 & "El Ingenio no quiere apoyar" \\
\hline 13 & $\begin{array}{l}\text { "El dinero a veces se tarda y hay que conseguir por otro lado } \\
\text { con más costo" }\end{array}$ \\
\hline 20 & "Los créditos nunca llegan a tiempo y jinetean el dinero" \\
\hline 23 & "Hay poca ganancia y mucha inversión" \\
\hline 24 & "El (Ingenio), solo financia su cultivo" \\
\hline 25 & "porque pide crédito" \\
\hline 36 & "Altos intereses de mi dinero" \\
\hline 44 & "Los créditos se tardan mucho y nuestro cultivo se atrasa" \\
\hline 50 & $\begin{array}{l}\text { "Las liquidaciones las pagan muy tarde y tengo que conseguir } \\
\text { para volver a financiar mi cultivo" }\end{array}$ \\
\hline 62 & "Intereses caros" \\
\hline 78 & $\begin{array}{l}\text { "Porque si pedimos crédito el interés es alto y no recibimos } \\
\text { apoyo del gobierno" }\end{array}$ \\
\hline 88 & $\begin{array}{l}\text { "Porque es poco y no alcanza para pagar riegos y porque es } \\
\text { riego por bombeo" }\end{array}$ \\
\hline 107 & "No me alcanza lo que gano para solventar el cultivo" \\
\hline 175 & "El precio es barato y apenas sale para los fertilizantes" \\
\hline 242 & "No llega a tiempo" \\
\hline
\end{tabular}

La tabla anterior muestra como algunos productores señalan los altos costos del fertilizante como su problemática, así como a la tardanza de los apoyos para solventar la implementación del cultivo.

Los resultados muestran de manera general como las Asociaciones Cañeras no presentaron como un problema para la mayoría de los productores de caña de azúcar, ya que el apoyo de estas organizaciones ha sido fundamental para que los productores lleven a buen fin el manejo de sus cosechas de caña de azúcar mediante la siembra y compra de su producto. Asimismo el manejo de los créditos por parte de estas Organizaciones con el otorgamiento de los mismos, han sido fluidos y expeditos en el momento de su solicitud. 


\section{Conclusiones}

El sentir de manera general fue positivo hacia las Organizaciones cañeras e Ingenio Melchor Ocampo. Ya que en cuestión de apoyos crediticios los productores manifestaron que estas asociaciones han sido importantes en la implementación de su cultivo, así como la entrega en tiempo y forma de los créditos solicitados. Asimismo manifiestan en su mayoría que en cuestión de asesoría agrícola la misma ha sido oportuna e expedita, por lo que no lo han visto como un problema para ellos.

Por otra parte señalan en más del $80 \%$ que los riegos hasta el momento han sido puntuales. No así la parte de fertilizantes que influye directamente en sus suelos, de los cuales señalan que cada día van más a la alza, mermando aún más su economía. Al respecto algunos de los problemas que manifiestan los agricultores de las dos principales organizaciones cañeras de este valle, es el agotamiento de sus suelos cosecha tras cosecha, así como el factor humedad, como principales problemas que se enfrentan año con año.

De manera general se puede mencionar que tanto las Asociaciones Cañeras e Ingenio Melchor Ocampo (IMO), no se presentaron como un problema para la mayoría de los productores de caña de azúcar, ya que el apoyo de estas organizaciones ha sido fundamental para que lleven a buen fin el manejo de sus cosechas de caña de azúcar. Asimismo el manejo de los créditos por parte de estas Asociaciones con el otorgamiento de los mismos, han sido fluidos y expeditos en el momento de su solicitud.

No queda más que agradecer en primera instancia a la Unión Nacional de Cañeros, A. C. CNPR (Confederación Nacional de la Propiedad Rural), la CNC (Confederación Nacional Campesina) y al Ingenio Melchor Ocampo (IMO), mediante sus presidentes: Ing. Gabriel F. Blackaller Ayala; Ing. José de Jesús Zúñiga Mendoza e Ing. Jesús Ernesto Ramos García respectivamente, por todo el apoyo brindado. Asimismo a los Ingenieros de la CNPR "Diego" y "Valdo" y muy especialmente a los productores de caña del valle de El Grullo-Autlán, quienes con su apoyo hicieron posible este proyecto. A TODOS MUCHAS 


\section{Bibliografía}

CONADESUCA. 2015. Comité Nacional para el Desarrollo Sustentable de la Caña de Azúcar. En: http://www.cndsca.gob.mx/

CNPR (2014). Informe interno. Zafra 2013-2014. Asociación de Agricultores del valle El Grullo-Autlán, A. C. octubre de 2014. Consulta restringida

Domínguez, R. L. (2005). Desarrollo regional y competitividad: La agroindustria azucarera en México. Revista de Ciencias Sociales y Humanidades. Universidad Autónoma de Ciudad Juárez. Ciudad Juárez Chihuahua. Enero-junio. Vol. 15. No. 027. Pp. 227-250.

Howard, G. (2013). ¿Un plan con caña? El paro de los 54 ingenios en todo el país parece ser un amargo pretexto para aumentar los precios del azúcar. Reporte Índigo. En: http://www.reporteindigo.com/

Malhotra, N.K. (2008). Investigación de Mercados. $5^{\mathrm{a}}$ Edición. Pearson Educación. México, D. F.

Quintero, G. S. D. (2003). Evaluación de impacto ambiental de la Agricultura en el Valle de Autlán, Jalisco. Tesis de Licenciatura. Universidad de Guadalajara. Centro Universitario de la Corta Sur. Ingeniero en Recursos Naturales y Agropecuarios. Págs. 154.

Riche S.; J. Bond; A. Jerardo; S. Haley. (2014). Sugar and Sweeteners Outlook. Economic Research Service Situation and Outlook. SSS-M-314. United States Department of Agriculture. October 17, 2014.

Sandoval L.; J. de J.; J. L. Sepúlveda T. (2012). Los beneficios del abono orgánico. La Gaceta. Universidad de Guadalajara. Año 11. Edición 705. Lunes 18 de junio. P. 14

Santos, P.J.; A. A. Muñoz; M. P. Juez y V. P. Cortiñas. (2003). Diseño de encuestas para estudios de mercado. Técnicas de muestreo y análisis multivariado. Centro de Estudios Ramón Aceves, S. A. Madrid, España.

ZAFRANET. (2015). Pesos por bulto de $50 \mathrm{Kg}$ en azúcar estándar en los últimos 12 meses (Oct 2014-Oct 2015). En: http://www.zafranet.com/ (Consultado el 30 de octubre de 2015) 